\title{
Design of Pressure Vessel Cylinder Based on
}

\section{Reliability Analysis}

\author{
Xingxu $\mathrm{Li}^{1, \mathrm{a}}$, Chenghong Duan ${ }^{1, \mathrm{~b}}$ \\ ${ }^{1}$ Beijing University of Chemical Technology, Beijing 100029, China \\ aLixingxu1@126.com, bduanchenghong@163.com
}

\begin{abstract}
Based on the JC method (reliability calculation method) proposed by the Lcwick et al. [1] and recommended by the Joint Committee on Structural Security (JCSS) and the fourth strength theory, the reliability design formula of the thin-walled cylinder of pressure vessel is deduced. And the Monte Carlo reliability calculation method of ANSYS is used to verify the correctness of the formula, and the factors that have the greatest influence on the structural reliability are analyzed, which can provide some reference for the design of pressure vessel reliability.
\end{abstract}

Keywords: JC method (reliability calculation method), reliability calculation formula, Monte Carlo, pressure vessel.

\section{Introduction}

With the petrochemical industry toward the trend of large-scale development, ensuring the safety of pressure vessels, and taking into account its economy has become increasingly important [2]. In the design of the pressure vessel, the design pressure, material strength and structural dimensions are all random, and the existing standards deal with these parameters in accordance, and using a larger safety factor to ensure the safety of structure, obviously this approach can not take into the safety and economy of pressure vessels. In this paper, the reliability formula of pressure vessel cylinder is deduced by the JC method (reliability calculation method) and the fourth strength theory. The Monte Carlo reliability calculation method of ANSYS is used to verify the structural reliability. The greatest influencing factors of structural reliability are analyzed, and provide reference for the reliability design of pressure vessel.

\section{Calculation formula of cylinder reliability}

Radial stress of thin-walled cylinder is much smaller than the axial stress and circumferential stress. According to the elastic mechanics, the three-way stress state of cylinder can be obtained as follows:

$$
\sigma_{1}=\frac{P D}{2 t}, \quad \sigma_{2}=\frac{P D}{4 t}, \quad \sigma_{3}=0
$$

In the formula (1), $P$ denotes the design pressure, $D$ denotes the inner diameter of cylinder, $t$ denotes the wall thickness of cylinder, and $\sigma_{i(i=1,2,3)}$ denotes the i-th 
principal stress.

According to the cylinder stress calculation formula and the fourth strength theory, we can see that the equivalent stress $\mathrm{S}$ is:

$$
S=\sqrt{\sigma_{1}^{2}-\sigma_{1} \sigma_{2}+\sigma_{2}^{2}}=\frac{\sqrt{3} P D}{4 t}
$$

$P, D$ and $t$ of formula (2) are treated as random variables. According to statistics and people's design experience, it can be assumed that these random variables conform to the normal distribution. At the same time, the Taylor formula expansion of $S$ is carried out at the mean of these variables, and more than one level of expand items are neglected. At the same time, the reliability formula of cylinder is calculated according to the functional function of cylinder (see equation (3) as follow:

$$
\beta=\frac{\mu_{R}-\mu_{S}}{\sqrt{\sigma_{R}^{2}+\sigma_{S}^{2}}} \frac{\mu_{R}-\frac{\sqrt{3} \mu_{P} \mu_{D}}{4 \mu_{t}}}{\sqrt{\sigma_{R}^{2}+\left(\frac{\sqrt{3} \mu_{D}}{4 \mu_{t}}\right)^{2} \sigma_{P}^{2}+\left(\frac{\sqrt{3} \mu_{P}}{4 \mu_{t}}\right)^{2} \sigma_{D}^{2}+\left(\frac{\sqrt{3} \mu_{P} \mu_{D}}{4 \mu_{t}^{2}}\right)^{2} \sigma_{t}^{2}}}
$$

According to formula (4), we can see the reliability of cylinder:

$$
P_{r}=\phi(\beta)
$$

The failure probability of cylinder is:

$$
P_{f}=1-P_{r}=\phi(-\beta)
$$

In the formula (3), $R$ is the yield strength of the material at the design temperature, taking 1.5 times the allowable stress at the design temperature as the yield stress of the material, $S$ is the equivalent stress of cylinder. In the formula (4), $\mu_{i(i=R, S, P, D, t)}$ is the mean value of the variable, $\sigma_{i(i=R, S, P, D, t)}^{2}$ is the variable variance, $\beta$ is the variable value of standard normal distribution function. In the equations (5) and (6), $\phi(x)$ is the probability cumulative distribution function of the standard normal distribution.

\section{3. cylinder design}

The design conditions of cylinder are shown in Table 1.

Table 1 Design conditions

\begin{tabular}{|c|c|c|}
\hline Parameter & Mean $\mu$ & Standard deviation $\sigma$ \\
\hline Design pressure (P/MPa) & 5 & 0.2 \\
\hline Design tempreture $\left(\mathrm{W} /{ }^{\circ} \mathrm{C}\right)$ & 100 & Note $^{1)}$ \\
\hline Inside diameter of cylinder $(\mathrm{D} / \mathrm{mm})$ & 1500 & 19.74 \\
\hline yield strength of material $(\mathrm{R} / \mathrm{MPa})$ & 282 & _ $^{\text {Note }}$ 1) \\
\hline Length of cylinder $(\mathrm{L} / \mathrm{mm})$ & 2000 & \\
\hline
\end{tabular}

Note 1: This variable is not a random variable.

According to the formula (4), the formula (5) and Table 1, repeated iterative method is used to determine the thickness of cylinder. The specific calculation steps are as follows: 
1) Assuming that the mean of cylinder thickness is $\mu_{t}=5$.

2) Assuming that the reliability of cylinder $P_{r}$, and checking the standard normal distribution table to obtain the variable value $\beta_{o}$.

3) Calculate the standard deviation $\sigma_{t}$ of cylinder thickness.

4) Putting the parameters in Table $1, \mu$ t and $\sigma$ into formula (4) to obtain $\beta$ of cylinder.

5) If $\beta \geq \beta o$, the thickness of cylinder meets the design requirements. Otherwise increasing the thickness of cylinder, and re-implementation (2) and (4) until $\beta \geq \beta$.

The standard deviation $\sigma \mathrm{t}$ of cylinder thickness is calculated as follows:

$$
\sigma_{t}=\mu_{t} C_{t}
$$

In the formula (7), $C_{t}$ is the variation factor of cylinder thickness. The value of $C_{t}$ is 0.0067 .

According to the experience of pressure vessel design, we can see the reliability of cylinder $P_{r}=99.999 \%$ [3]. Checking the standard normal distribution function table shows, variation $\beta_{o}=4.27$. According to the calculation steps 1 ) to 5), the calculation results of cylinder thickness see Table 2 .

Table 2 Calculation results of cylinder thickness

\begin{tabular}{|c|c|c|}
\hline Part & Mean $\mu_{t}$ & Standard deviation $\sigma_{t}$ \\
\hline The thinkness of cylinder $(\mathrm{t} 1 / \mathrm{mm})$ & 18 & 0.1206 \\
\hline
\end{tabular}

\section{Reliability analysis of ANSYS}

The finite element model shown in Fig. 1 is established by ANSYS. The reliability analysis of cylinder structure is carried out. Defining the function function as follow:

$$
Z=R-\max S
$$

In the formula (8), maxS represents the maximum von Mises stress.

Using Monte Carlo method of ANSYS to obtain the failure probability Pf of structure, that is the probability of $Z \leq 0$.

According to the ANSYS reliability analysis results, we can see:

1 ) in the case of $95 \%$ confidence, the probability of $\mathrm{Z}<0$ is $0.078 \%$, that is the failure probability of structure is $0.078 \%$, so the reliability of the structure is 99.922\%.

2) In the sampling process of functional function $Z$ (see Fig. 2), the mean convergence, indicating that the number of simulation enough.

3) The sensitivity analysis of output variable $Z$ (functional function) is shown in Fig. 3. hrough the results of sensitivity analysis we can see that $R, P$ and $t 1$ have a great influence on the functional function, and the influence of the other parameters is small. When $R$ increase, the carrying capacity of the structure increases and the reliability increases. 


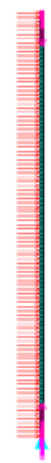

Fig. 1 Finite element model of cylinder
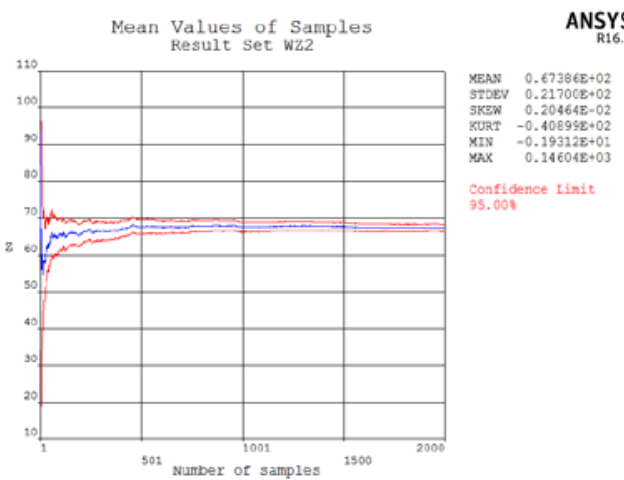

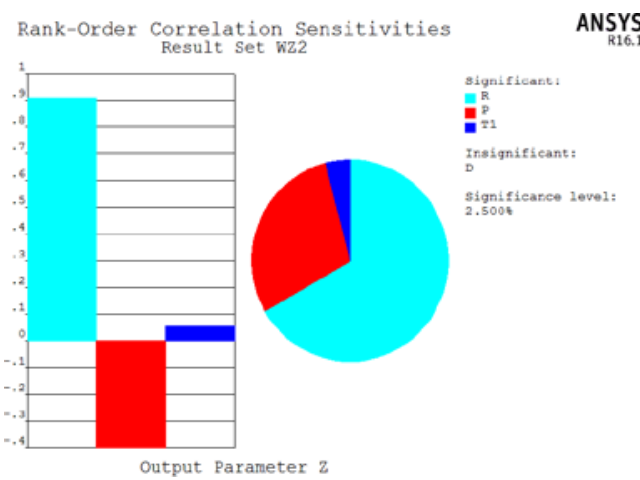

Fig. 3 Sensitivity analysis of functional function $Z$

\section{Conclusion}

In this paper, the formula of reliability design of pressure vessel cylinder is deduced by JC method, and the thickness of cylinder is calculated by $99.999 \%$ reliability. The reliability analysis is carried out by Monte Carlo method of ANSYS. The result of ANSYS calculation is that the reliability of cylinder is $99.922 \%$ under the condition of $95 \%$ confidence level, which is very close to the theoretical formula 99.999\% reliability. Therefore, the reliability formula of this paper can be used to design the reliability of pressure vessel cylinder.

\section{Acknowledgments}

This work was financially supported by the fund of The National Research and Development Program of China (2016YFB1100202-1).

The author of the communication is Chenghong Duan.

\section{References}

[1]. Rackwitz, R. and B. Flessler, Structural reliability under combined random load sequences. Computers \& Structures, 1978. 9(5): p. 489 - 494.

[2]. Zhiming Lu, Reliability design of pressure vessel and its application on solid rocket motor vessel, 2009, Beijing Jiaotong University. p. 121.

[3]. Yan Wang, Design method and application researching of pressure vessel Based on reliability theory. Science and technology information, 2010(35): p. 500-523. 